\title{
Selecting representative ages for developmental changes of respiratory irregularities and hypoxic ventilatory response in rats
}

\author{
Lalah M Niane*, Aida Bairam
}

Unité de Recherche en Périnatologie, Centre Hospitalier Universitaire de Québec, Hôpital Saint-François d'Assise, Département de Pédiatrie, Université Laval, Québec.

Email: *lalah-malika.niane.1@ulaval.ca

Received 9 April 2011; revised 26 April 2011; accepted 5 May 2011.

\begin{abstract}
Apnea frequency and the weak ventilatory response to hypoxia are a major clinical correlates of the immaturity of respiratory control system in preterm neonates. Rats are frequently used as model to study the respiratory control during development. However, little is known about the postnatal ages that best represent these respiratory irregularities and the hypoxic ventilatory response. Using plethysmography, we assessed baseline minute ventilation, ventilatory response to moderate hypoxia $\left(\mathrm{FiO}_{2}=12 \%, 20 \mathrm{~min}\right)$ and apnea frequency in awake and non-anesthetized rats at the postnatal ages of $1,4,7,12,21$ and 90 days old (P1, P4, P7, P12, P21, and P90, respectively). Baseline minute ventilation slightly increased in P4 ( $25 \%$ vs $P 1)$ then gradually decreased with age (age effect: $p<0.05)$. The lowest level of ventilation was observed in $P 90$ ( $p<0.01$ vs all ages). Minute ventilation ( $\%$ from baseline) in response to hypoxia showed the well-known biphasic pattern in all rats at 12 days old or less. Minute ventilation at the initial phase of the hypoxic response was not significantly different between P1, P4, between P7, P12 and between P21, P90. The late phase of the hypoxic response was similar between P1, P4, and between P21, P90, but was significantly different between P7 and P12 (p < 0.05). Under baseline or hypoxic condition, the higher number of apnea frequency (spontaneous and postsigh) was observed in P1, it then decreased progressively with age (age effect: $p<0.01$ for baseline; $p<$ 0.001 for hypoxia). These results suggest that when P4, P7 and P12 are selected to represent the age-dependent changes of the hypoxic ventilatory response in rats, the $P 1$ rats should be included to better describe the age-dependence of apnea frequency.
\end{abstract}

Keywords: Apnea; Hypoxia; Newborn Rat

\section{INTRODUCTION}

The respiratory control system evolves rapidly in newborn mammals during the neonatal period. The major clinical correlates of the immaturity of this system are periodic breathing and apnea, particularly in preterm neonates. Accordingly, apnea in preterm neonates is systematically treated. The hypoxic ventilatory response (HVR) is regularly used to evaluate the immaturity of the respiratory control system. This response is not fully developed at birth and undergoes significant changes during the postnatal period. In newborn mammals, the HVR presents as a biphasic pattern consisting of an initial increase in ventilation, resulting from a stimulation of peripheral chemoreceptors (primarily located in the carotid bodies), followed by a decline, in some cases to a level below pre-hypoxic levels. It is likely that the decline in ventilation originates centrally and ultimately overrides the excitatory input from peripheral chemoreceptors. The maturation of the HVR during the postnatal period may take days to weeks depending on the mammalian species studied [1-3]. Although the developmental pattern of the respiratory control system has been described in multiple newborn animal models, such as mice, rat, cat, rabbit, piglet, and lambs, longitudinal studies assessing ventilation in awake and non-anesthetized rats are few [4,5]. Indeed, respiratory irregularities have yet to be assessed in a population of rats from birth to adult ages. Using rats as an animal model, our aim was to determine the frequency of spontaneous apnea, as an index of respiratory irregularities, and to concurrently evaluate the HVR, as an index of maturation of the chemoreceptor reflex to hypoxia in specific postnatal age groups and as adult. The secondary aim was to select ages that could longitudinally reflect the development of the respiratory control system as well as reduce the age-population of rat pups used for these types of invest- 
tigations. We choose rats for the following reasons: the central nervous system of rat pups born at term is immature in comparison with humans and has been roughly compared to that of infants born at $\sim 28$ weeks of gestation [6]. Indeed, the HVR of rat pups at 3 - 5 days old is similar to that observed in preterm humans below 30 weeks of gestational age; at about 2 weeks old, it is similar to full term human neonates [1]. Finally, the maturation of the central nervous system as a whole in rats occurs during the first three weeks of age [6], thus providing a highly reliable and practical model to determine the developmental pattern of the HVR at a very specific postnatal age. Using plethysmography, we recorded baseline ventilation and the HVR by exposing rats to moderate hypoxia $\left(\mathrm{FiO}_{2}=12 \%, 20 \mathrm{~min}\right)$. We assessed the frequency of apnea during the baseline and the steady state of hypoxic response. Rats at ages $1,4,7$, 12 , and 21 days old and adults at 90 days old were studied.

\section{MATERIALS AND METHODS}

\subsection{Animals}

The local Animal Care Committee at Laval University approved the experimental protocol. The study was performed on 94 Sprague-Dawley male rat pups born in our animal care facility from 14 virgin females and males. At birth (P1), litters were culled to 12 pups with a preference for males. Because steroidal sex hormones may affect the HVR [7], we preferred to use males to ensure the homogeneity of our study at all ages. Those that were used at adulthood ( 90 days old) were weaned from their mother at 21 days old and were placed 2 per cage to be studied at 90 days old.

\subsection{Plethysmography Recording}

Respiratory and metabolic indexes were recorded in P1 (12-24 h following birth; $\mathrm{n}=9), \mathrm{P} 4(\mathrm{n}=20), \mathrm{P} 7(\mathrm{n}=13)$, P12 ( $\mathrm{n}=17)$, P21 $(\mathrm{n}=24)$ rats using a whole body flow-through plethysmograph (IOX, Emka Technologies, Paris, France) as we regularly used in newborn rats $[8,9]$. The gas flow through the plethysmograph was set at 100 $\mathrm{ml} / \mathrm{min}$ for P1-P7 rats and $200 \mathrm{ml} / \mathrm{min}$ for P12 and P21 rats (flowmeter, model 4140; TSI, Shoreview, MN). The temperature inside the plethysmograph was set at $34^{\circ} \mathrm{C}$ $(\mathrm{P} 1), 32^{\circ} \mathrm{C}(\mathrm{P} 4$ and $\mathrm{P} 7)$ and $30^{\circ} \mathrm{C}(\mathrm{P} 12)$ using a temperature-control system (Physitemp, Clifton, NJ, USA), and the relative humidity was continuously measured from the out-flowing air stream. In P90 rats $(n=11)$, ventilation was recorded using a double-chamber plethysmograph (model PLY 3023, Buxco Electronics, Sharon, CT, USA) where the gas flows through the front (head) and rear chambers were set at about 100 and $500 \mathrm{ml} / \mathrm{min}$, respectively[10]. The respiratory flow tracing, recorded using the rear chamber of the plethysmography, was calibrated by injecting a known volume of air into the chamber $[9,10]$. The inflow and outflow of oxygen were continuously recorded using a dedicated oxygen analyzer (AEI Technologies; Naperville, IL, USA). Body temperature was measured via the mouth in $\mathrm{P} 1, \mathrm{P} 4$ and P7 rats or via the rectum for the older rats before and at the end of the experiment using a thermocouple for small or adult rodents (Harvard, Holliston, MA, USA). Respiratory frequency and tidal volume were recorded from the plethysmograph signal. Tidal volume was first corrected depending on barometric pressure, room and body temperature, and humidity (BTPS) using the Bartlett and Tenney equation [11], and afterward, it was used to calculate minute ventilation (respiratory frequency $\mathrm{X}$ tidal volume). Because the aim of this study was to describe the developmental pattern of minute ventilation in response to hypoxia, the changes in respiratory frequency and tidal volume during development are not presented for brevity. As an index of metabolism, oxygen consumption was calculated as follows: flow $\times$ $\left[\left(\mathrm{O}_{2, \text { in }}-\mathrm{O}_{2, \text { out }}\right)-\mathrm{O}_{2, \text { out }} \times\left(\mathrm{CO}_{2, \text { out }}-\mathrm{CO}_{2 \text {,in }}\right)\right] /\left(1-\mathrm{O}_{2, \text { out }}\right)$. However, the calculation was corrected to STPD conditions as had been done previously [9,10]. All details for the sources of apparatus were set as reported previously [8-10,12].

\subsection{Baseline Ventilation and Hypoxic Response}

Each rat studies only once and was placed into the plethysmograph at least 20 - 30 min before experiment for adaptation. After the body temperature measurement, baseline normoxic variables $\left(\mathrm{FiO}_{2}=21 \%\right)$ were recorded for $10 \mathrm{~min}$. Then, the pup was exposed to moderate hypoxia $\left(\mathrm{FiO}_{2}=12 \%, 20 \mathrm{~min}\right)$ by mixing pure nitrogen with air flowing through the plethysmograph to achieve $12 \% \mathrm{O}_{2}$ concentration in approximately $2 \mathrm{~min}$. These first $2 \mathrm{~min}$ were not considered for calculation. At the end of the recording, the body temperature was immediately remeasured.

\subsection{Apnea Frequency}

The frequency of apnea was calculated during the last 10 min of baseline recording and the last $10 \mathrm{~min}$ of hypoxic response (steady state) using the standardized criteria of Mendelson [13], as we have used previously [8,12,14]. Two types of apnea were selected. Spontaneous apnea was defined as the interruption of flow for at least two normal respiratory cycles, and post-sigh apnea occurred when the breath amplitude was at least twice the resting tidal volume [13] (See examples, Figures 4(a) and (b)). Because all newborn rats showed a very low frequency of post- sigh apnea (about 10\%) during baseline, in accordance with previous studies [14] but a very low 
spontaneous apneas (about 15\%) during hypoxia, we combined both types of apnea (total apnea) under each conditions studied. Respiration is largely dependent on the sleep stage [15]. Although we did not record the sleep-wake state during recording, the rats were closely observed, and if necessary we gently knocked on the wall of the plethymograph to keep them awake.

\subsection{Data Collection and Statistical Analyses.}

The ventilatory variables collected on a minute-byminute basis by IOX software (Version 1.8.9 EMKA technology, Paris, France) were averaged over the last 5 $\min$ for the baseline values, every 2 min between $2-10$ min for the initial phase of the hypoxic response and every $5 \mathrm{~min}$ for the last $10 \mathrm{~min}$, during the late phase of the hypoxic response. Oxygen consumption was measured at the baseline and at the end of the hypoxic response. A one-way ANOVA was used to compare different ages, and a p-value $<0.05$ was considered significant. Data are presented as mean $\pm \mathrm{SEM}$.

\section{RESULTS}

\subsection{Baseline Minute Ventilation and Metabolism}

Minute ventilation increased slightly at $\mathrm{P} 4$ compared with P1 as has been previously observed [5]. Minute ventilation was then gradually decreased with age and the lowest level was observed in P90 (adult) rats as compared with all of the other ages studied (Figure 1(a)). Interestingly, the level of minute ventilation observed in adult rats was comparable to a previous study that used a similar double plethysmography chamber [10] and with a study that used a whole body plethysmograph room [12]. Oxygen consumption decreased with age corresponding with a higher metabolic rate in newborns compared with adults. Then, it decreased progressively following the pattern of minute ventilation decreasing with age. Oxygen consumption was not different between P1 and P4 or between P7, P12 and P21. The lowest oxygen consumption was observed in $\mathrm{P} 90$ compared with all other ages (Figure 1(b)).

\subsection{HVR Across Ages}

Because of the difference in baseline ventilation across ages studied (Figure 1(a)), the HVR was expressed as the percentage of increase from the baseline. The HVR was biphasic in all pups less or equal to 12 days old (Figure 2). At 4 min of HVR, minute ventilation was significantly higher than baseline in all ages studied ( $\mathrm{P}$ varied from 0.01 to 0.001 ; for clarity, the labels are not included on the figures). Its level was similar between $\mathrm{P} 1$ and P4, between P7 and P12, and between P21 and P90 (Figure 2). During the late phase of HVR (at $20 \mathrm{~min}$

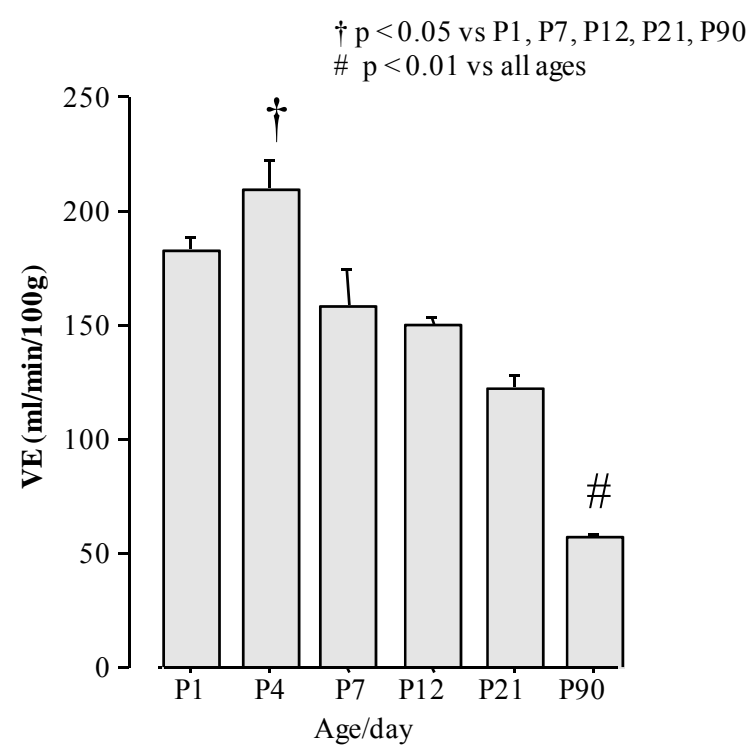

(a)

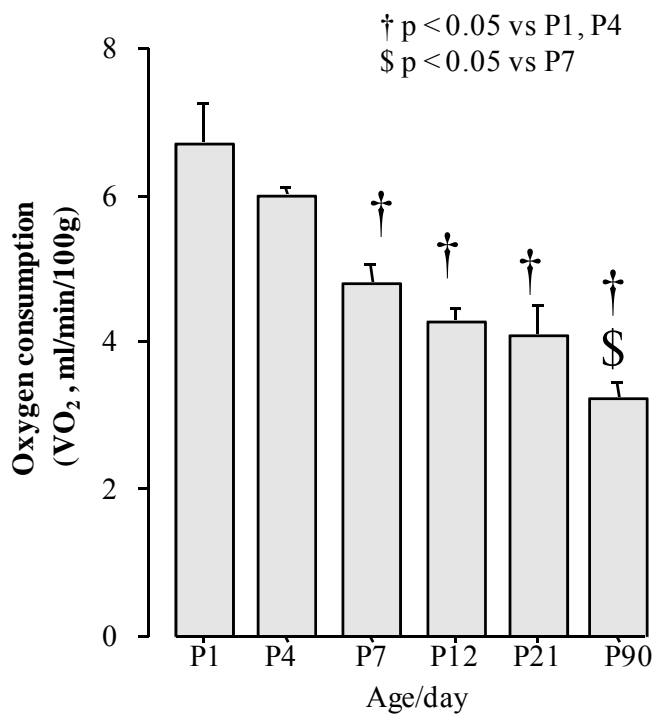

(b)

Figure 1. Baseline minute ventilation (a) and metabolism (b) across ages studied in rats. Data are means \pm SEM.

of hypoxia), minute ventilation was significantly lower than the baseline in P1 and P4 ( $<<0.05)$, not different from the baseline in $\mathrm{P} 7$ and significantly higher than the baseline in $\mathrm{P} 12(\mathrm{p}<0.05), \mathrm{P} 21$ and $\mathrm{P} 90$ rats $(\mathrm{p}<0.0001$, for clarity the labels are not included in the figures). Again, its level was not different between P1 and P4 but was lower than P7 (Figure 2). In P12, minute ventilation at 20 min of HVR was higher than P7 but lower than P21 and $\mathrm{P} 90$ rats. Finally, neither the initial nor the late phase of HVR was different between P21 and P90 rats (Figure 2). At the end of HVR, the lowest oxygen consumption 

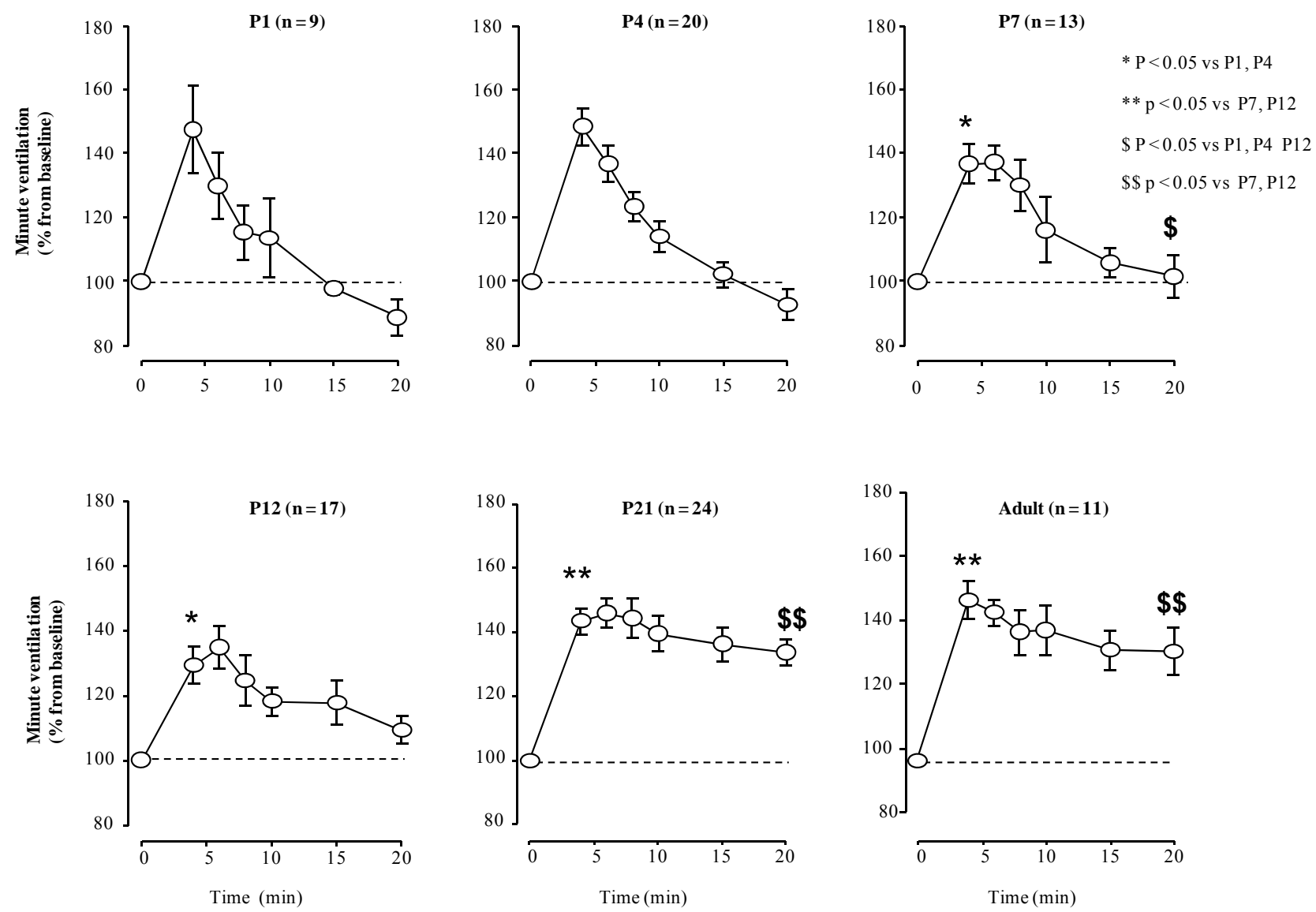

Figure 2. Minute ventilation in response to moderate hypoxia $\left(\mathrm{FiO}_{2}=12 \%, 20 \mathrm{~min}\right)$ across ages studied in rats. Minute ventilation is expressed as percentage change from the baseline. Data are means $\pm \mathrm{SEM}$.

was observed in P12 as compared with all other ages (Figure 3).

\subsection{Apnea Frequency across Ages.}

Figure 4 showed examples of spontaneous (A) and postsigh (B) apnea recorded in P12 rats. The occurrence of apnea was significantly higher during hypoxia than that observed during baseline (Figures 4(c) and (d)) in each of age studied ( $\mathrm{p}$ values were: $0.001,0.005,0.008,0.009$, 0.02, 0.01 for P1, P4, P7, P12, P21 and P90, respectively). Apnea frequency decreased gradually with age (Figures 4(c) and (d)) showing a highly significant age-dependent correlation, whether the $\mathrm{P} 90$ rats were included or not during baseline $\left(\mathrm{R}^{2}=-0.599\right.$; Correlation $\left.\mathrm{p}<0.0001\right)$ or hypoxia $\left(\mathrm{R}^{2}=-0.605\right.$; Correlation $\left.\mathrm{p}<0.0001\right)$.

\section{DISCUSSION}

The respiratory control system undergoes intense developmental changes during postnatal life $[1,2,16]$. One major question that is debated regularly is what are the most appropriate ages of animal models that best reflect the developmental pattern of respiratory control. On the basis of our current results, we suggest that P4, P7 and

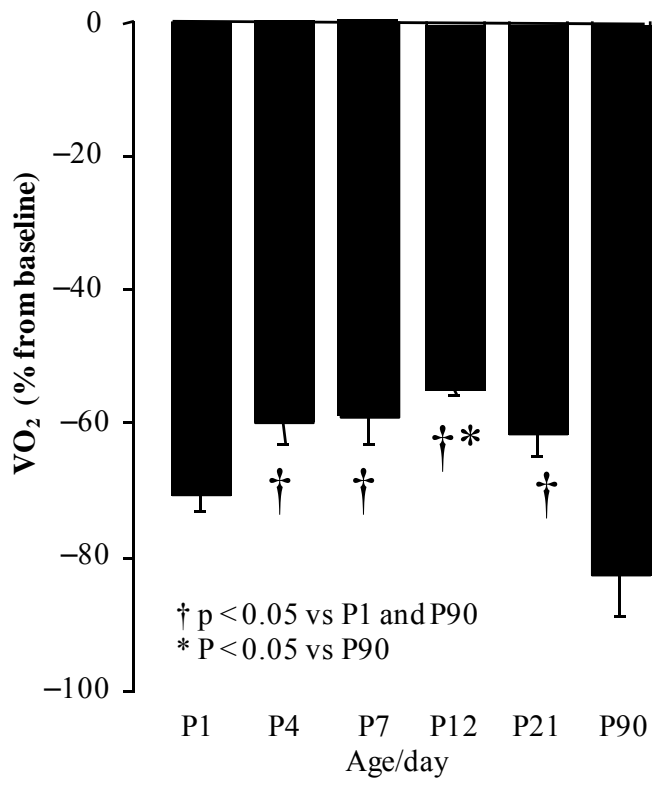

Figure 3. Oxygen consumption at the end of hypoxic exposure across ages studied in rats. Oxygen consumption is expressed as percentage change from the baseline. Data are means \pm SEM. 


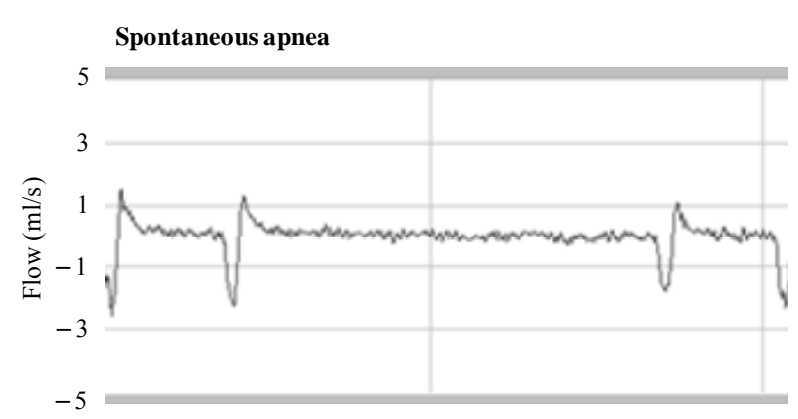

(a)

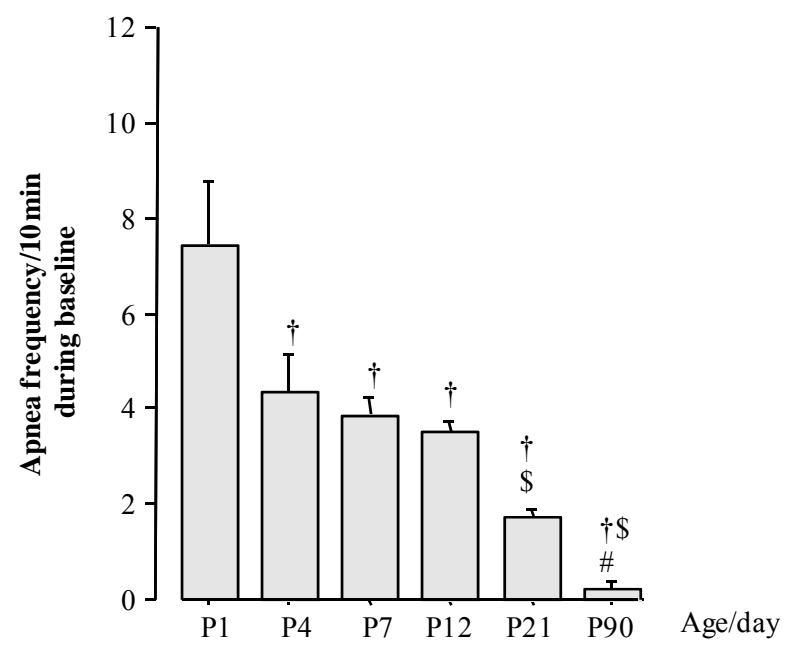

(c)

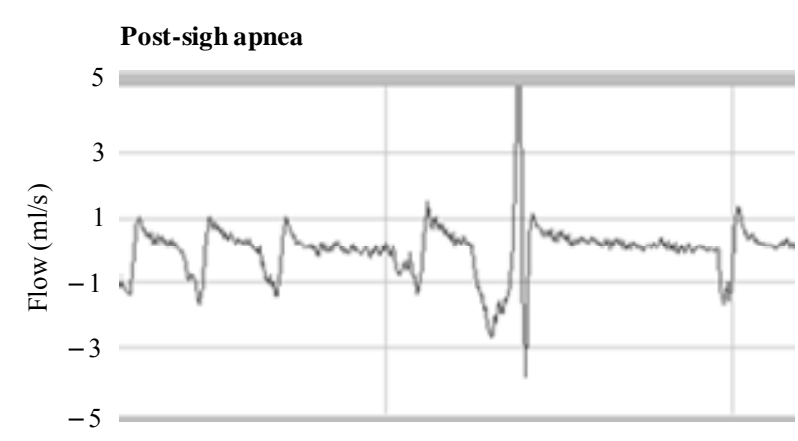

(b)

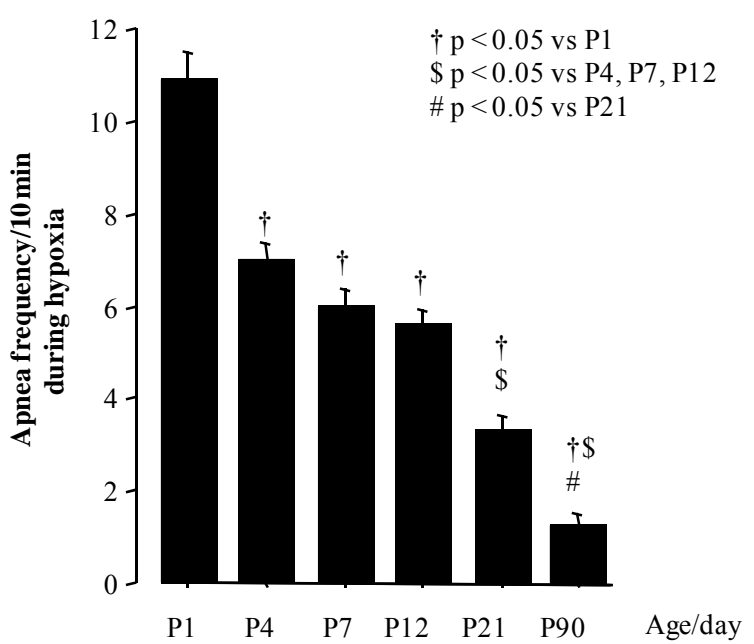

(d)

Figure 4. Examples of spontaneous (a) and post-sigh (b) apnea recorded during last $10 \mathrm{~min}$ of baseline and hypoxia in P12 rats. Total apnea frequency during baseline (c) and hypoxia (d) across ages studied in rats. Data are means \pm SEM.

clinical correlation of these observations, we further suggest that P4 rats may be representative of very immature babies of less than 28 weeks of gestation, P7 rats of immature babies less than 36 weeks of gestation and P12 rats to term babies $[1,3,17]$. However, the P1 rats may need to be considered when studying respiratory irregularities such as apnea.

Two main studies have described the effects of age on the HVR to hypoxia in newborn rats using whole body plethysmography in awake and non-anesthetized newborn rats; however, in either study adult rats are not included. Liu et al. [5] focused on the changes in the respiratory pattern cycle in rats from birth to 21 days old while Eden et al. [4] mainly studied the HVR to different levels of inspired oxygen. For example, in Liu et al., the same rat was tested at different postnatal ages to assess the HVR during 5 min exposures to $10 \%$ oxygen. In Eden et al., the same rat was also used at different postnatal ages and was exposed in each experiment to multiple levels of inspired oxygen, 8,12 , and $15 \%$. In our experimental design we recorded each rat just once, be- cause repeated handling [18] and repeated exposure to stimuli [19] may disrupt the developmental process of the respiratory control system in a newborn, and such disruption may persist well into adult life [2,20,21]. Indeed, the level of hypoxia was moderate but sustained for $20 \mathrm{~min}$, allowing us better evaluate the developmental pattern of the biphasic HVR and metabolism. It was clear that while the late phase progressively increased with age, the initial phase showed different relationships with age. P1 and P4 rats significantly increased their ventilation in response to hypoxia, although this increase was for a very short duration. Later, at P7 and P12, the level of the initial increase of ventilation was lower than that observed in younger rats, but it was more sustained. Hence, we proposed that ages of P4, P7 and P12 could be representative of the developmental aspect of both the peripheral and central components of the HVR. Finally, as indices of respiratory irregularities during the neonatal period we counted apnea events during the baseline and the steady state of hypoxic response. Under each condition, the frequency of apnea was highly dependent 
on age providing a useful index of immaturity of the respiratory control system. It is suggested that apnea frequency can be used as an efficient parameter to study respiratory irregularities in rats and an additional variable to study the development of respiratory control.

One pitfall related to the use of whole body plethysmography is the accuracy of tidal volume measurements. Despite this limitation, this method remains the best available for measuring ventilatory variables (including tidal volume) in awake, unrestrained small animals $[11,22,23]$. As discussed previously [8,9,14,12], all experiments were conducted under similar conditions, and the tidal volume was corrected by considering barometric pressure, humidity in the plethysmograph, body temperature and body weight. Conversely, ventilation is affected by sleep state [15], and rats during the first 15 days of life spend about $70 \%$ of their time asleep [24,25]. However, rats were carefully observed during recording, and if they showed signs of falling asleep, we gently knocked on the wall of the recording chamber to keep the rat awake [12,14].

In conclusion, respiratory irregularities such as apnea and periodic breathing are frequently observed in preterm infants. These irregularities are related to an immaturity of the breathing control system [17], and rats are regularly used as a model. We propose that rats at 4,7 and 12 days old could be used to study the developmental pattern of mechanisms, factors or drugs that affect the HVR. However, rats at P1 old would be included to better describe the age-dependence of apnea frequency.

\section{ACKNOWLEDGEMENTS}

This study was supported, in part, by the CIHR operating grant MOP81101 to A. Bairam. We thank Mrs. Melanie Pelletier and Sylvie Viger for animal care.

\section{REFERENCES}

[1] Bissonnette, J.M. (2000) Mechanisms regulating hypoxic respiratory depression during fetal and postnatal life. American Journal of Physiology Regulatory, Integrative and Comparative Physiology, 278, R 1391-1400.

[2] Carroll, J.L. (2003) Developmental plasticity in respiratory control. Journal of Applied Physiology, 94, 375-389.

[3] Cohen, G. and Katz-Salamon, M. (2005) Development of chemoreceptor responses in infants. Respiratory Physiology \& Neurobiology, 149, 233-242.

doi:10.1016/j.resp.2005.02.013

[4] Eden, G.J. and Hanson, M.A. (1987) Maturation of the respiratory response to acute hypoxia in the newborn rat. Journal of Physiology, 392, 1-9.

[5] Liu, Q., Lowry, T.F. and Wong-Riley, M.T. (2006) Postnatal changes in ventilation during normoxia and acute hypoxia in the rat: implication for a sensitive period. Journal of Physiology, 577, 957-970. doi:10.1113/jphysiol.2006.121970
[6] Romijn, H.J., Hofman, M.A. and Gramsbergen, A. (1991) At what age is the developing cerebral cortex of the rat comparable to that of the full-term newborn human baby? Early Human Development, 26, 61-67. doi:10.1016/0378-3782(91)90044-4

[7] Behan, M. and Wenninger, J.M. (2008) Sex steroidal hormones and respiratory control. Respiratory Physiology \& Neurobiology, 164, 213-221.

doi:10.1016/j.resp.2008.06.006

[8] Julien, C., Bairam, A. and Joseph, V. (2008) Chronic intermittent hypoxia reduces ventilatory long-term facilitation and enhances apnea frequency in newborn rats. American Journal of Physiology Regulatory, Integrative and Comparative Physiology, 294, R1356-1366. doi:10.1152/ajpregu.00884.2007

[9] Niane, L.M., Donnelly, D.F., Joseph, V. and Bairam, A. (2010) Ventilatory and carotid body chemoreceptor responses to purinergic $\mathrm{P} 2 \mathrm{X}$ receptor antagonists in newborn rats. Journal of Applied Physiology, 110, 83-94. doi:10.1152/japplphysiol.00871.2010

[10] Julien, C.A., Niane, L., Kinkead, R., Bairam, A. and Joseph, V. (2010) Carotid sinus nerve stimulation, but not intermittent hypoxia, induces respiratory LTF in adult rats exposed to neonatal intermittent hypoxia. American Journal of Physiology Regulatory, Integrative and Comparative Physiology, 299, R192-205. doi:10.1152/ajpregu.00707.2009

[11] Bartlett, D.Jr. and Tenney, S.M. (1970) Control of breathing in experimental anemia. Respiratory Physiology, 10, 384-395. doi:10.1016/0034-5687(70)90056-3

[12] Montandon, G., Bairam, A. and Kinkead, R. (2006) Long-term consequences of neonatal caffeine on ventilation, occurrence of apneas, and hypercapnic chemoreflex in male and female rats. Pediatric Research, 59, 519-524. doi:10.1203/01.pdr.0000203105.63246.8a

[13] Mendelson, W.B., Martin, J.V., Perlis, M., Giesen, H., Wagner, R. and Rapoport, S.I. (1988) Periodic cessation of respiratory effort during sleep in adult rats. Physiology \& Behavior, 43, 229-234. doi:10.1016/0031-9384(88)90243-0

[14] Julien, C.A., Joseph, V. and Bairam, A. (2010) Caffeine reduces apnea frequency and enhances ventilatory long-term facilitation in rat pups raised in chronic intermittent hypoxia. Pediatric Research, 68, 105-111. doi:10.1203/PDR.0b013e3181e5bc78

[15] Parmeggiani, P.L. (1985) Regulation of circulation and breathing during sleep: experimental aspects. Annals of Clinical Research, 17, 185-189.

[16] Putnam, R.W., Conrad, S.C., Gdovin, M.J., Erlichman, J.S. and Leiter, J.C. (2005) Neonatal maturation of the hypercapnic ventilatory response and central neural $\mathrm{CO}_{2}$ chemosensitivity. Respiratory Physiology \& Neurobiology, 149, 165-179. doi:10.1016/j.resp.2005.03.004

[17] Darnall, R.A., Ariagno, R.L. and Kinney, H.C. (2006) The late preterm infant and the control of breathing, sleep, and brainstem development: a review. Clinics in Perinatology, 33, 883-914. doi:10.1016/j.clp.2006.10.004

[18] Gulemetova, R. and Kinkead, R. (2011) Neonatal stress increases respiratory instability in rat pups. Respiratory Physiology \& Neurobiology, in press. doi:10.1016/j.resp.2011.01.014

[19] Mateika, J.H. and Narwani, G. (2009) Intermittent hy- 
poxia and respiratory plasticity in humans and other animals: does exposure to intermittent hypoxia promote or mitigate sleep apnoea? Experimental Physiology, 94, 279-296. doi:10.1113/expphysiol.2008.045153

[20] Bavis, R.W. and Mitchell, G.S. (2008) Long-term effects of the perinatal environment on respiratory control. Journal of Applied Physiology, 104, 1220-1229. doi:10.1152/japplphysiol.01086.2007

[21] Montandon, G., Bairam, A. and Kinkead, R. (2008) Neonatal caffeine induces sex-specific developmental plasticity of the hypoxic respiratory chemoreflex in adult rats, American Journal of Physiology Regulatory, Integrative and Comparative Physiology, 295, R922-934. doi:10.1152/ajpregu.00059.2008

[22] Enhorning, G., Schaik, van S., Lundgren, C. and Vargas, I. (1998) Whole-body plethysmography, does it measure tidal volume of small animals? Canadian Journal of Physiology and Pharmacology, 76, 945-951.

doi:10.1139/y99-002

[23] Mortola, J.P. and Frappell, P.B. (1998) On the barometric method for measurements of ventilation, and its use in small animals. Canadian Journal of Physiology and Pharmacology, 76, 937-944. doi:10.1139/y99-001

[24] Blumberg, M.S., Seelke, A.M., Lowen, S.B. and Karlsson, K.A. (2005) Dynamics of sleep-wake cyclicity in developing rats. Proceedings of the National Academy of Sciences, USA, 102, 14860-14864. doi:10.1073/pnas.0506340102

[25] Frank, M.G. and Heller, H.C. (1997) Development of REM and slow wave sleep in the rat. American Journal of Physiology, 272, R1792-1799. 